\title{
Functional Characterization of RNA-Binding Protein Isolated from Hot Pepper
}

\author{
Hyun Min Kim, Mi Jin Jeon, Seo Young Park, Sang Hoon Ma, Young Hee Joung \\ School of Biological Sciences and Technology, Chonnam National University \\ 77 Yongbong-Ro, Gwangju 61185, Republic of Korea \\ tblinj@naver.com; jmj8745@ naver.com: seoyoung302@naver.com; kingmasalo@naver.com; yhjoung@jnu.ac.kr
}

\section{Extended Abstract}

Solanaceae plants such as tomato and pepper show a sympodial growth architecture. In sympodial growth, the development of a shoot apical meristem is terminated by a flower or an inflorescence, and further growth continues from the uppermost axillary meristems. Finally, a short shoot segment known as the sympodial unit, consisting of three leaves and an inflorescence in tomato, and two leaves and a solitary flower in pepper, is reiterated in the life cycle of Solanaceae. Flowering time in members of the Solanaceae plant family is an important agronomic trait for controlling shoot architecture and improving yield. To investigate the feasibility of flowering time regulation, an RNA-binding protein (CaRBP) was isolated from hot pepper (Capsicum annuum cv. Bukang). RBPs are very important regulators in a variety of cellular processes including splicing, nuclear export, polyadenylation, RNA stability, decay, and translation. The deduced amino acid sequence showed most similarity to the human nucleolar protein NIFK. Human NIFK protein interacts with the forkhead-associated domain of Ki-67 protein in a mitosis-specific and phosphorylation dependent manner, suggesting that this protein functions in cell cycle progression. CaRBP was highly expressed in the vegetative and reproductive tissues, such as leaves and fruits, respectively. Subcellular localization analysis indicated that CaRBP is a nucleolar protein. To investigate the function of the CaRBP using a transgenic approach, a full-length CaRBP ORF under the control of 35S promoter was constructed. This construct was then introduced into tomato plants (Solanum lycopersicum cv. Micro-Tom). Under long-day conditions (16-h light/8-h dark), transgenic plants exhibited markedly delayed flowering, thereby leading to continuous vegetative growth over one year. Thus, they did not show any inflorescence, compared to wild-type plants. They also showed a dwarf phenotype and severe morphological defects of the vegetative organs such as curled leaves and non-serrated. This floral retardation was associated with the alteration of SFT (Single Flower Truss) and SOC1 (Suppressor of Overexpression of Constans1) as floral integrators. In addition, CaRBP negatively controls COLs (Constans-Like) via modulations in the expression of CDF3, FLOWERING BHLH, FLAVIN-BINDING, KELCH REPEAT and F-BOX1 (SIFKF1). This indicates a repressive effect of CaRBP on the regulation of flowering time in tomato. Overall, these results suggest that alteration in CaRBP expression levels may provide an effective means of controlling flowering time in tomato plant. 